\title{
Initiation of any tobacco and five tobacco products across 3 years among youth, young adults and adults in the USA: findings from the PATH Study Waves 1-3 (2013-2016)
}

\author{
Cassandra A Stanton 주, ,'2 Eva Sharma 주 , ${ }^{1}$ Elizabeth L Seaman, ${ }^{1}$ \\ Karin A Kasza (1) , Kathryn C Edwards (1), , Michael J Halenar (1), \\ Kristie A Taylor (1) , ${ }^{1}$ Hannah Day, ${ }^{4}$ Gabriella Anic, ${ }^{4}$ Lynn C Hull, ${ }^{4}$ \\ Maansi Bansal-Travers (1) , ${ }^{3}$ Jean Limpert, ${ }^{4}$ Lisa D Gardner (1) , ${ }^{4}$ Hoda T Hammad, ${ }^{4}$ \\ Nicolette Borek, ${ }^{4}$ Heather L Kimmel $10,{ }^{5}$ Wilson M Compton, ${ }^{5}$ Andrew Hyland ${ }^{3}$
}

\begin{abstract}
- Additional material is published online only. To view please visit the journal online (http://dx.doi.org/10.1136/ tobaccocontrol-2019-055573).
\end{abstract}

'Behavioral Health and Health Policy Practice, Westat, Rockville, MD, United States 2Department of Oncology, Georgetown University Medical Center, Washington, DC, United States

${ }^{3}$ Department of Health Behavior, Roswell Park Comprehensive Cancer Center, Buffalo, NY, United States

${ }^{4}$ Office of Science, Center for Tobacco Products, Food and Drug Administration, Silver Spring, MD, United States ${ }^{5}$ National Institute on Drug Abuse, National Institutes of Health, Bethesda, MD, United States

\section{Correspondence to} Dr Cassandra A Stanton, Behavioral Health and Health Policy, Westat, Rockville, MD 20850, USA;

cassandrastanton@westat.com

Received 16 December 2019 Revised 7 February 2020 Accepted 10 February 2020

\section{ABSTRACT}

Objective This study reports weighted cross-sectional prevalence of never use of tobacco, and longitudinal past 12-month (P12M), past 30-day (P30D) and frequent P30D any tobacco or specific tobacco product initiation across three 1-year waves. Longitudinal three-wave pathways are examined to outline pathways of exclusive and polytobacco initiation, as well as pathways of new initiators of electronic nicotine delivery systems (ENDS) or cigarettes.

Design Data were drawn from the first three waves (2013-2016) of the Population Assessment of Tobacco and Health Study, a nationally representative, longitudinal cohort study of US youth and adults. Respondents with data at all three waves (youth, $\mathrm{N}=11$ 046; young adults, $N=6478$; adults $25+, N=17$ 188) were included in longitudinal analyses.

Results Across the three age groups, weighted cross-sectional analyses revealed never any tobacco use decreased each year from 2013 to 2016, reflecting overall increases in tobacco initiation in the population during this time. Compared with cigarettes, cigars, hookah and smokeless tobacco, ENDS had the highest proportion of $\mathrm{P} 12 \mathrm{M}$ initiation from Wave 1 to Wave 3 (W3) for each age group. Among youth Wave 2 P30D initiators of exclusive ENDS or cigarettes, the most common W3 outcome was not using any tobacco (ENDS: $59.0 \%$ (95\% Cl 48.4 to 68.8 ); cigarettes: $40.3 \%$ (95\% Cl 28.7 to 53.1$)$ )

Conclusions Initiation rates of ENDS among youth and young adults have increased the number of ever tobacco users in the US prevention strategies across the spectrum of tobacco products which can address youth initiation of tobacco products.

\section{INTRODUCTION}

(C) Author(s) (or their employer(s)) 2020. No commercial re-use. See rights and permissions. Published by BMJ.

To cite: Stanton CA

Sharma E, Seaman EL, et al. Tob Control

2020;29:s178-s190.
National estimates of tobacco use in the US have demonstrated a decline in ever cigarette use in the past two decades; lifetime cigarette use among 12th graders was down to $23.8 \%$ in 2018 , compared with $63.1 \%$ in 1991, according to Monitoring the Future data. ${ }^{1}$ Lifetime smokeless tobacco use among 12 th graders was down to $10.1 \%$ in 2018 , compared with $32.4 \%$ in 1992 , demonstrating a similar pattern. ${ }^{1}$ Ever use of electronic nicotine delivery systems (ENDS), which in this report refers largely to e-cigarettes, has increased in recent years among youth (12-17 years). ${ }^{2-8}$ The 2019 National Youth Tobacco Survey results indicate that $27.5 \%$ of US high school students are current users of e-cigarettes and 5.8\% are current cigarette smokers. ${ }^{8}$ Among adults, data from the Population Assessment of Tobacco and Health (PATH) Study indicate that $11 \%$ of young adults (18-24 years) initiated current tobacco use over a 1 -year period from Wave 1 (W1) in 2013/2014 to Wave 2 (W2) in 2014/2015, compared with only 4\% initiation of current tobacco use between W1 and W2 among adults $25+$ (ages 25 and older). ${ }^{9}$

Initiation of tobacco use can be defined broadly as new ever use, ${ }^{10-13}$ or in reference to a specific time period such as past 12 -month use (P12M), ${ }^{11} 14$ past 30-day use (P30D) ${ }^{10} 13$ or frequent use (eg, 20 or more days) in the past 30 days. ${ }^{15} 16$ These increasingly narrow definitions for new use can help distinguish when new use may be a function of experimentation (ie, P12M use) or more regular use (ie, frequent use in the past 30 days). Reports of any tobacco initiation have traditionally been driven by cigarette use ${ }^{4}$; however, recent increases in use of non-cigarette tobacco products calls for monitoring initiation of other tobacco products among existing tobacco users (eg, cigarette smokers who initiate ENDS). ${ }^{17} 18$

It has been shown that most people who ever smoked daily try their first cigarette during youth or young adulthood, with very few $(<1.5 \%)$ initiating cigarette use after age $26 .{ }^{4}$ Cross-sectional 2016 data from National Survey on Drug Use and Health (NSDUH) report retrospective P12M initiation rates of cigarettes, smokeless tobacco and cigars for youth at 3.2\%, $1.5 \%$ and $2.4 \%$, respectively, and for adults ages 18 and older at 1.1\%, $0.4 \%$ and $1.1 \%$, respectively. ${ }^{14}$ Moreover, P30D daily cigarette smoking in the $\mathrm{P} 12 \mathrm{M}$ is higher among youth compared with adults, as shown by cross-sectional NSDUH rates that were tracked from 2006 to $2013 .{ }^{16}$

It is important to understand patterns of any tobacco initiation among never tobacco users and 
new product initiation among existing tobacco users because a robust literature suggests that early cigarette smoking initiation, independent of sociodemographics, increases the risk of experiencing smoking-related morbidities and all-cause mortality later in life. ${ }^{19}$ An analysis of W1 and W2 PATH Study data found that among youth participants, any use of ENDS, hookah, noncigarette combustible tobacco or smokeless tobacco was associated with cigarette initiation over the next year, and that polytobacco use increased the odds of cigarette initiation. ${ }^{10}$ Several studies examining initiation of ENDS use have reported that ENDS initiation among never cigarette smokers may influence the initiation of cigarette smoking among youth. ${ }^{72} 21$ The many ways in which ever and current tobacco product use can shape future tobacco product initiation and progression are important to track in longitudinal studies that can monitor how these patterns impact addiction liability and potential negative health outcomes.

The current study draws from the nationally representative, longitudinal data of the PATH Study to follow participants' tobacco use over three waves of data collection (2013/14, $2014 / 15$ and 2015/16). The availability of these longitudinal data allows a detailed examination over time of W1 (2013/14) never any tobacco or never specific-tobacco-product users to follow initial uptake and transitions across 2 years at Wave 2 (W2; 2014/15) and W3 (2015/16). Our first aim is to report weighted cross-sectional prevalence of never tobacco use (for any tobacco and individual tobacco products: cigarettes, ENDS, cigars, hookah and smokeless tobacco) among youth, young adults and adults $25+$. The second aim is to report weighted P12M, P30D and frequent (20 or more days) P30D initiation rates among never users for each age group across 2 years (W1W3) and compare initiation rates of the products in each of the 1 year intervals (W1-W2, W2-W3). The third aim is to test age group differences in weighted longitudinal W1-W2-W3 P30D exclusive and polytobacco use initiation pathways among W1 never tobacco users. Given that recent studies have linked ENDS use and subsequent new cigarette smoking among youth, $71020-24$ the fourth aim is to descriptively explore the subgroups of W2 ENDS initiation and cigarette initiation to better understand pathways among initiators of these products such as continued use, discontinued use and switching at W3.

\section{METHODS}

\section{Study design and population}

The PATH Study is an ongoing, nationally representative, longitudinal cohort study of youth (ages 12-17) and adults (ages 18 or older) in the US. Self-reported data were collected using audio computer-assisted self-interviews administered in English and Spanish. The PATH Study recruitment employed a stratified address-based, area-probability sampling design at W1 that oversampled adult tobacco users, young adults (ages 18-24) and African American adults. An in-person screener was used at W1 to randomly select youth and adults from households for participation in the study. At W1, the weighted response rate for the household screener was 54.0\%. Among screened households, the overall weighted response rate was $78.4 \%$ for youth and $74.0 \%$ for adults at W1, $87.3 \%$ for youth and $83.2 \%$ for adults at W2, and $83.3 \%$ for youth and $78.4 \%$ for adults at W3. Further details regarding the PATH Study design and W1 methods are published elsewhere. ${ }^{25} 26$ Details on interview procedures, questionnaires, sampling and weighting and information on accessing the data are available at https://doi.org/10.3886/Series606. The study was conducted by Westat and approved by the Westat Institutional Review Board. All participants ages 18 and older provided informed consent, with youth participants ages $12-17$ providing assent while their parent/legal guardian provided consent.

Online supplementary figure 1 shows the full W1 sample respondents who have data at Waves 1,2 and 3. The current analysis reports cross-sectional estimates from 13651 youth and 32320 adults who participated in W1 data collection (12 September 2013 through 14 December 2014); or 12172 youth and 28362 adults at W2 (23 October 2014 through 30 October 2015); or 11814 youth and 28148 adults at W3 (19 October 2015 to 23 October 2016). The differences in number of completed interviews between W1, W2 and W3 reflect attrition due to non-response, mortality and other factors, as well as youth who enrol in the study at W2 or W3 ${ }^{25}$ We report on initiation estimates in all age groups: longitudinal W1-W2 data include participants in W1 who were also in W2 (youth, $\mathrm{n}=11$ 996; young adults, $n=7324$; adults $25+, n=19$ 116); W2-W3 data include participants in W2 who were also interviewed at W3 (youth, $\mathrm{n}=11$ 279; young adults, $\mathrm{n}=7252$; adults $25+$, $n=18$ 129). We also report longitudinal estimates for participants with data at all three waves (youth, $n=11046$; young adults, $n=6478$; adults $25+, n=17$ 188). Youth included those who were youth at all three waves $(n=7595)$ and those who were youth at W1 and aged up to the young adult group at W2 $(n=1714)$ or W3 $(n=1737)$. The young adults included those who were young adults at all three waves $(n=4590)$ or aged up to the adults $25+$ group in W2 $(n=949)$ or W3 $(n=939)$.

\section{Measures}

Tobacco use

At each wave, adults and youth were asked about their tobacco use behaviours for cigarettes, ENDS (which includes e-cigarettes), traditional cigars, cigarillos, filtered cigars, pipe tobacco, hookah, snus pouches, other smokeless tobacco (loose snus, moist snuff, dip, spit or chewing tobacco) and dissolvable tobacco. Respondents were asked about 'e-cigarettes' at W1 and 'e-products' (ie, e-cigarettes, e-cigars, e-pipes and e-hookah) at W2 and W3; for the purposes of this paper, all electronic products noted above are referred to as ENDS. In addition, youth were asked about their use of bidis and kreteks. However, use of bidis, kreteks and dissolvable tobacco was not included in the analyses due to small sample sizes.

\section{Outcome measures}

Never use: never use of any tobacco and each individual product (cigarettes, ENDS, hookah, cigar, and smokeless tobacco) is defined as not having ever used the product/any tobacco even once or twice.

Initiation of use: P12M initiation is defined as never use at W1 and any tobacco/tobacco product use at follow-up for a specific tobacco product independent of other tobacco product use. P30D initiation is defined as never use at W1 and any tobacco/ tobacco product use within the past 30 days at follow-up, and frequent P30D initiation is defined as never use at W1 and any tobacco/tobacco product use within the past 30 days on at least 20 or more days at follow-up. Each stricter timeframe for initiation is a subset of the larger timeframe preceeding it. Duration of follow-up is either 1 or 2 years depending on the aim. See table footnotes for more details. P30D exclusive initiation is defined as never tobacco use at W1 and new use of only one tobacco product in the past 30 days at a subsequent wave. P30D polytobacco initiation is defined as never tobacco use at W1 and new use of two or more tobacco products in the past 30 days at a subsequent wave (W2 or W3). Longitudinal outcomes examined 
also include P30D initiation of exclusive or polytobacco ENDS or cigarettes at W2 among W1 never users.

\section{Analytic approach}

To address Aim 1, weighted cross-sectional prevalence of never any tobacco use and never use of each individual product was estimated at each wave, stratified by age group. For Aim 2, weighted P12M, P30D and frequent P30D initiation rates for any tobacco and the individual tobacco products for each age group from $\mathrm{W} 1$ to $\mathrm{W} 2, \mathrm{~W} 2$ to $\mathrm{W} 3$ and $\mathrm{W} 1$ to $\mathrm{W} 3$ were estimated. To address Aim 3, longitudinal W1-W2-W3 P30D exclusive initiation and polytobacco initiation pathways among W1 never users were examined. For Aim 4, transitions in P30D tobacco use by W3 among W1 never tobacco users who initiated ENDS or cigarette use at W2 are reported separately.

Cross-sectional estimates (Aim 1) were calculated using PATH Study cross-sectional weights for W1 and single-wave (pseudocross-sectional) weights for W2 and W3. The weighting procedures adjusted for complex study design characteristics and non-response. Combined with the use of a probability sample, the weighted data allow these estimates to be representative of the non-institutionalised, civilian, resident US population aged 12 or older at the time of each wave. Longitudinal estimates
(Aims 2, 3 and 4) were calculated using the PATH Study W3 all-waves weights. These weighted estimates are representative of the resident US population aged 12 and older at the time of W3 (other than those who were incarcerated) who were in the civilian, non-institutionalised population at W1. For Aims 1-3, weighted t-tests were conducted on differences in proportions to assess statistical significance. To correct for multiple comparisons, Bonferroni post-hoc tests were conducted.

All analyses were conducted using SAS Survey Procedures, V.9.4 (SAS Institute). Variances were estimated using the balanced repeated replication method ${ }^{27}$ with Fay's adjustment set to 0.3 to increase estimate stability. ${ }^{28}$ Analyses were run on the W1-W3 Public Use Files (https://doi.org/10.3886/ICPSR36498.v8). Estimates based on fewer than 50 observations in the denominator or with a coefficient of variation greater than 0.30 were flagged.

\section{RESULTS}

\section{Cross-sectional weighted prevalence}

Prevalence estimates of never any tobacco and product-specific never use at W1, W2 and W3 by each age group (youth, young adults and adults $25+$ ) are shown in figure 1 . There were relatively modest, but statistically significant, decreases across waves

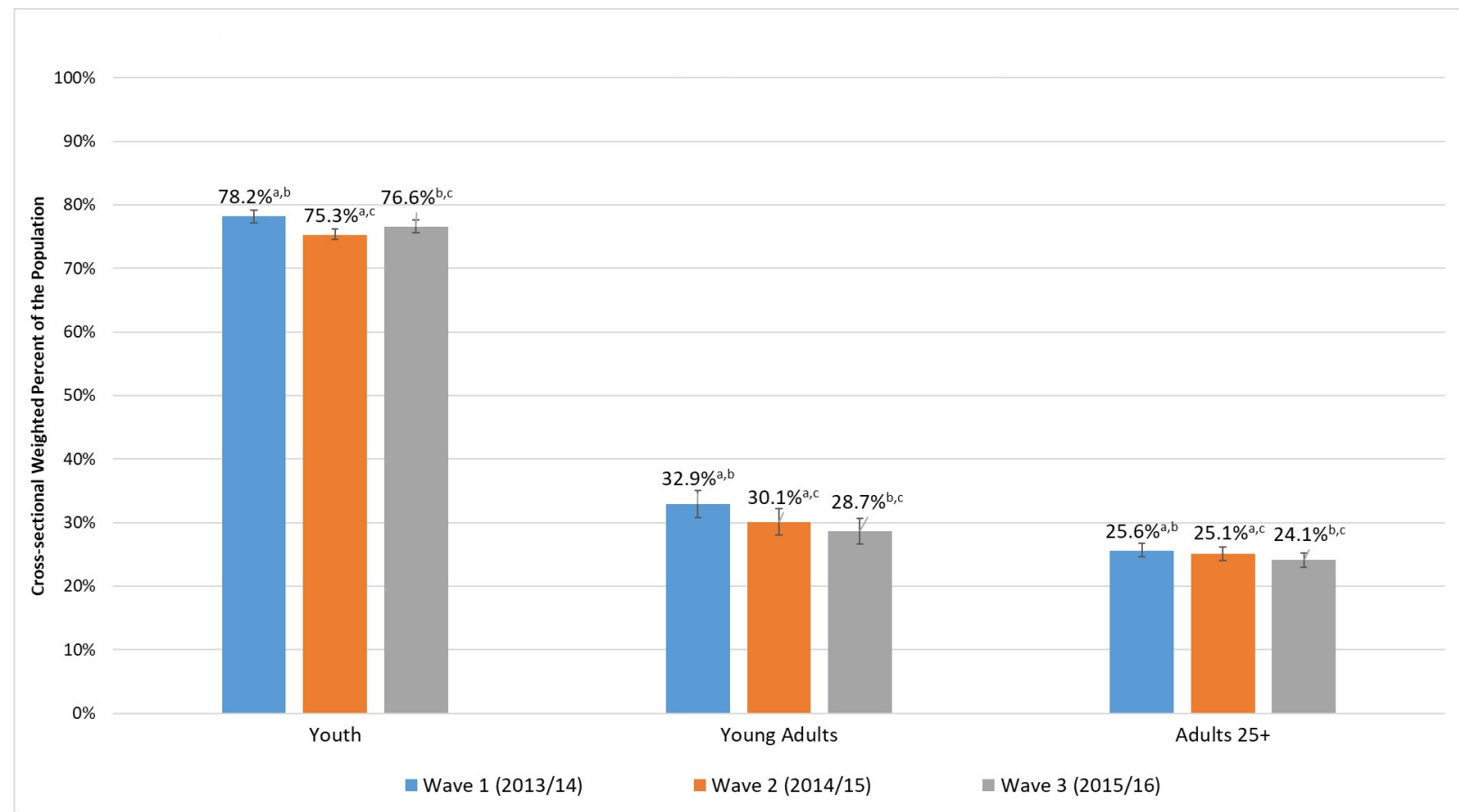

Figure 1 Cross-sectional weighted percentages of never tobacco use among youth, young adults and adults 25+ in W1, W2 and W3 of the PATH Study.

W1 = Wave 1; W2 = Wave 2; W3 = Wave 3; PATH = Population Assessment of Tobacco and Health

W1/W2/W3 never tobacco use unweighted Ns: youth (ages 12-17) = 10 246/8721/8487; young adults (ages 18-24) = 1755/1850/2096; adults 25+ (ages 25 and older) $=2761 / 2323 / 2193$.

X-axis shows three age groups: youth, young adults and adults 25+. Y-axis shows weighted percentages of W1, W2 and W3 never users in these three age groups. Sample analysed includes all W1, W2 and W3 respondents at each wave. The PATH Study cross-sectional (W1) or single-wave weights (W2 and W3) were used to calculate estimates at each wave. All respondents with data at one wave are included in the sample for that wave's estimate and do not need to have complete data at all three waves. Never tobacco use is defined as not having used any tobacco, even once or twice in lifetime.

aSignificant difference at $\mathrm{p}<0.0167$ (Bonferroni corrected for three comparisons) between W1 and W2.

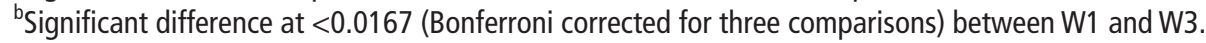

'Significant difference at $<0.0167$ (Bonferroni corrected for three comparisons) between W2 and W3.

The logit-transformation method was used to calculate the $95 \% \mathrm{Cls}$.

Analyses were run on the W1, W2 and W3 public use files (https://doi.org/10.3886/ICPSR36498.v8). 
Table 1a Weighted Percentage of Tobacco and Product-Specific Initiation at W2 or W3 Among W1 Never Users

\begin{tabular}{|c|c|c|c|c|c|c|}
\hline \multirow{3}{*}{$\begin{array}{l}\text { W1 (2013/14) } \\
\text { Youth }\end{array}$} & \multicolumn{6}{|c|}{ W2 $(2014 / 15)$ or W3 $(2015 / 16)$} \\
\hline & \multicolumn{2}{|c|}{ P12M Initiation } & \multicolumn{2}{|c|}{ P30D Initiation } & \multicolumn{2}{|c|}{ Frequent P30D Initiation } \\
\hline & Weighted \% & $95 \% \mathrm{Cl}$ & Weighted \% & $95 \% \mathrm{Cl}$ & Weighted \% & $95 \% \mathrm{Cl}$ \\
\hline Never tobacco use & 24.4 & $(23.4-25.4)$ & 9.5 & $(8.8-10.2)$ & 1.3 & $(1.1-1.6)$ \\
\hline Never cigarette use & $9.9^{\mathrm{a}, \mathrm{d}}$ & $(9.3-10.6)$ & $4.7^{a, b, d}$ & $(4.2-5.3)$ & $0.6^{\mathrm{b}, \mathrm{c}}$ & $(0.5-0.8)$ \\
\hline Never ENDS* use & $22.7^{\mathrm{a}, \mathrm{e}, \mathrm{fg}}$ & $(21.6-23.7)$ & $6.4^{\mathrm{a}, \mathrm{e}, \mathrm{f}, \mathrm{g}}$ & $(5.8-7.1)$ & $0.9^{e, f, g}$ & $(0.7-1.2)$ \\
\hline Never hookah use & $8.9^{\mathrm{e}, \mathrm{i}}$ & $(8.3-9.7)$ & $3.4^{\mathrm{b}, \mathrm{e}, \mathrm{i}}$ & $(3.0-3.8)$ & $0.2^{b, e, i}$ & $(0.1-0.4)$ \\
\hline Never cigar use & $9.0^{f j}$ & $(8.3-9.7)$ & $4.0^{\mathrm{fj} j}$ & $(3.7-4.4)$ & $0.1^{c, f_{j, j}}$ & $(0.1-0.2)$ \\
\hline Never smokeless use & $4.2^{d, g, i, i j}$ & $(3.7-4.7)$ & $1.8^{\mathrm{d}, \mathrm{g}, \mathrm{ij}}$ & $(1.6-2.1)$ & $0.5^{9, i, j}$ & $(0.4-0.7)$ \\
\hline Young adults & Weighted \% & $95 \% \mathrm{Cl}$ & Weighted \% & $95 \% \mathrm{Cl}$ & Weighted \% & $95 \% \mathrm{Cl}$ \\
\hline Never tobacco use & 22.8 & $(20.0-26.0)$ & 12.2 & $(10.1-14.6)$ & 1.7 & $(1.1-2.7)$ \\
\hline Never cigarette use & $10.3^{a, b, d}$ & $(8.8-12.0)$ & $6.1^{d}$ & $(5.1-7.4)$ & $1.1^{\mathrm{b}, \mathrm{c}}$ & $(0.7-1.6)$ \\
\hline Never ENDS use & $28.4^{a, e, f, g}$ & $(26.5-30.4)$ & $7.4^{9}$ & $(6.4-8.4)$ & $1.0^{\mathrm{e}, \mathrm{fg}}$ & $(0.7-1.5)$ \\
\hline Never hookah use & $14.1^{\mathrm{b}, \mathrm{e}, \mathrm{i}}$ & $(12.5-15.8)$ & $6.4^{i}$ & $(5.3-7.7)$ & $0.2 t^{b, e}$ & $(0.1-0.4)$ \\
\hline Never cigar use & $12.0^{\mathrm{fj} j \mathrm{j}}$ & $(10.8-13.4)$ & $7.2^{\mathrm{j}}$ & $(6.2-8.4)$ & $0.3 t^{c, f}$ & $(0.2-0.6)$ \\
\hline Never smokeless use & $2.5^{d, g, i, i j}$ & $(2.1-2.9)$ & $1.4^{\mathrm{d}, \mathrm{g}, \mathrm{i}, \mathrm{j}}$ & $(1.1-1.7)$ & $0.4^{9}$ & $(0.2-0.6)$ \\
\hline Adults $25+$ & Weighted \% & $95 \% \mathrm{Cl}$ & Weighted \% & $95 \% \mathrm{Cl}$ & Weighted \% & $95 \% \mathrm{Cl}$ \\
\hline Never tobacco use & 4.0 & (3.1-5.0) & 2.7 & $(2.0-3.6)$ & $0.4 t$ & $(0.2-0.9)$ \\
\hline Never cigarette use & $2.4^{\mathrm{a}, \mathrm{d}}$ & $(1.8-3.2)$ & $1.8^{\mathrm{b}, \mathrm{d}}$ & $(1.3-2.6)$ & $0.4 \dagger$ & $(0.2-0.7)$ \\
\hline Never ENDS use & $6.7^{\mathrm{a}, \mathrm{e}, \mathrm{f}, \mathrm{g}}$ & $(6.3-7.2)$ & $2.3^{e, g}$ & $(2.0-2.5)$ & $0.5^{e, g}$ & $(0.4-0.6)$ \\
\hline Never hookah use & $1.4^{\mathrm{e}, \mathrm{h}, \mathrm{i}}$ & $(1.2-1.7)$ & $0.7^{b, e, h}$ & $(0.6-0.9)$ & $0.0^{\mathrm{e}, \mathrm{h}}$ & $(0.0-0.1)$ \\
\hline Never cigar use & $3.3^{f, h, j}$ & $(2.8-3.9)$ & $2.1^{\mathrm{h}, \mathrm{j}}$ & $(1.8-2.6)$ & $0.3^{\mathrm{h}, \mathrm{j}}$ & $(0.2-0.5)$ \\
\hline Never smokeless use & $0.7^{\mathrm{d}, \mathrm{g}, \mathrm{ij} j}$ & $(0.5-0.8)$ & $0.5^{\mathrm{d}, \mathrm{g}, \mathrm{j}}$ & $(0.4-0.7)$ & $0.1 t^{g, j}$ & $(0.0-0.1)$ \\
\hline
\end{tabular}

W1 total group unweighted denominators (P12M initiation/P30D initiation/Frequent P30D initiation):

Never tobacco use - youth (ages 12-17): 8,245/8,143/8,078; young adults (ages 18-24): 1,312/1,308/1,295; adults $25+$ (ages 25 and older): 2,043/2,046/2,039

Never cigarette use - youth: 9,596/9,590/9,576; young adults: 2,310/2,310/2,294; adults 25+:2,903/2,906/2,894

Never ENDS use - youth: 9,849/9,793/9,764; young adults: 3,697/3,651/3,639; adults 25+: 11,532/11,403/11,431

Never hookah use - youth: 10,239/10,231/10,199; young adults: 2,921/2,924/2,920; adults 25+: 13,071/13,083/13,065

Never cigar use - youth: 9,916/9,876/9,849; young adults: 2,817/2,818/2,770; adults 25+: 7,494/7,510/7,444

Never smokeless use - youth: 10,252/10,248/10,236; young adults: 5,145/5,152/5,135; adults 25+: 13,116/13,155/13,094

Analysis included youth, young adult, and adult 25+ W1 never tobacco or tobacco product users with data at W1, W2, and W3. Respondent age was calculated based on age at W1. W3 longitudinal (all-waves) weights were used to calculate estimates.

Never tobacco/product initiation is defined as not having used any tobacco or the specific product, even once or twice in lifetime.

1) P12M initiation: Defined as a never user at W1 who used any tobacco or the specific product within the past 12 months at W2 or W3.

2) P30D initiation (a subset of P12M initiation): Defined as a never user at W1 who used any tobacco or the specific product within the past 30 days at W2 or W3.

3) Frequent P30D initiation (a subset of P30D initiation): Defined as a never user at W1 who used any tobacco or the specific product within the past 30 days on at least 20 or more days at $\mathrm{W} 2$ or $\mathrm{W} 3$.

All significance tests were done between product initiations only (not any tobacco initation).

${ }^{a}$ denotes significant difference at $p<0.005$ (Bonferroni corrected for ten comparisons) between cigarette and ENDS

${ }^{b}$ denotes significant difference at $p<0.005$ (Bonferroni corrected for ten comparisons) between cigarette and hookah

'denotes significant difference at $p<0.005$ (Bonferroni corrected for ten comparisons) between cigarette and cigar

denotes significant difference at $p<0.005$ (Bonferroni corrected for ten comparisons) between cigarette and smokeless

edenotes significant difference at $p<0.005$ (Bonferroni corrected for ten comparisons) between ENDS and hookah

${ }^{f}$ denotes significant difference at $p<0.005$ (Bonferroni corrected for ten comparisons) between ENDS and cigar

${ }^{9}$ denotes significant difference at $p<0.005$ (Bonferroni corrected for ten comparisons) between ENDS and smokeless

hdenotes significant difference at $p<0.005$ (Bonferroni corrected for ten comparisons) between hookah and cigar

'denotes significant difference at $p<0.005$ (Bonferroni corrected for ten comparisons) between hookah and smokeless

'denotes significant difference at $p<0.005$ (Bonferroni corrected for ten comparisons) between cigar and smokeless

*Respondents were asked about "e-cigarettes" at W1 and "e-products" (i.e., e-cigarettes, e-cigars, e-pipes, and e-hookah) at W2 and W3

The logit-transformation method was used to calculate the $95 \% \mathrm{Cls}$.

†Estimate should be interpreted with caution because it has low statistical precision. It is based on a denominator sample size of less than 50, or the coefficient of variation of the estimate or its complement is larger than $30 \%$.

Analyses were run on the W1, W2, and W3 Public Use Files (https://doi.org/10.3886/ICPSR36498.v8).

$\mathrm{Cl}$, confidence interval; ENDS, electronic nicotine delivery system; P30D, past 30-day; P12M, past 12-month; W1, Wave 1; W2, Wave 2; W3, Wave 3.

Table 1b Weighted Percentage of Tobacco and Product-Specific Initiation at W2 Among W1 Never Users

\begin{tabular}{|c|c|c|c|c|c|c|}
\hline \multirow{3}{*}{$\begin{array}{l}\text { W1 (2013/14) } \\
\text { Youth }\end{array}$} & \multicolumn{6}{|c|}{ W2 (2014/15) } \\
\hline & \multicolumn{2}{|c|}{ P12M Initiation } & \multicolumn{2}{|c|}{ P30D Initiation } & \multicolumn{2}{|c|}{ Frequent P30D Initiation } \\
\hline & Weighted $\%$ & $95 \% \mathrm{Cl}$ & Weighted $\%$ & $95 \% \mathrm{Cl}$ & Weighted $\%$ & $95 \% \mathrm{Cl}$ \\
\hline Never tobacco use & 15.0 & $(14.2-15.8)$ & 5.3 & (4.7-5.8) & 0.7 & $(0.5-0.9)$ \\
\hline Never cigarette use & $4.6^{\mathrm{a}, \mathrm{d}}$ & $(4.2-5.1)$ & $2.1^{\mathrm{a}, \mathrm{d}}$ & $(1.8-2.4)$ & $0.4^{\mathrm{c}}$ & $(0.3-0.5)$ \\
\hline
\end{tabular}

Continued 
Table 1b Continued

\begin{tabular}{|c|c|c|c|c|c|c|}
\hline \multirow{3}{*}{$\begin{array}{l}\text { W1 }(2013 / 14) \\
\text { Youth }\end{array}$} & \multicolumn{6}{|c|}{ W2 (2014/15) } \\
\hline & \multicolumn{2}{|c|}{ P12M Initiation } & \multicolumn{2}{|c|}{ P30D Initiation } & \multicolumn{2}{|c|}{ Frequent P30D Initiation } \\
\hline & Weighted \% & $95 \% \mathrm{Cl}$ & Weighted \% & $95 \% \mathrm{Cl}$ & Weighted \% & $95 \% \mathrm{Cl}$ \\
\hline Never ENDS* use & $15.8^{\mathrm{a}, \mathrm{e}, \mathrm{f}, \mathrm{g}}$ & $(15.0-16.7)$ & $3.4^{a, e f, f g}$ & $(2.9-3.8)$ & $0.5^{\mathrm{e}, \mathrm{f}}$ & $(0.3-0.7)$ \\
\hline Never hookah use & $5.3^{\mathrm{e}, \mathrm{i}}$ & $(4.9-5.8)$ & $2.0^{e, i}$ & $(1.8-2.3)$ & $0.2^{\mathrm{e}}$ & $(0.1-0.3)$ \\
\hline Never cigar use & $4.6^{\mathrm{fj} j}$ & $(4.2-5.1)$ & $2.0^{\mathrm{fj} j}$ & $(1.8-2.3)$ & $0.1^{c, f, j}$ & $(0.1-0.2)$ \\
\hline Never smokeless use & $2.2^{\mathrm{d}, \mathrm{g}, \mathrm{ij} j}$ & $(1.9-2.5)$ & $0.9^{\mathrm{d}, \mathrm{g}, \mathrm{i}, \mathrm{j}}$ & $(0.7-1.1)$ & $0.3^{j}$ & $(0.2-0.4)$ \\
\hline Young adults & Weighted \% & $95 \% \mathrm{Cl}$ & Weighted \% & $95 \% \mathrm{Cl}$ & Weighted \% & $95 \% \mathrm{Cl}$ \\
\hline Never tobacco use & 15.2 & $(12.9-17.8)$ & 8.1 & $(6.5-10.0)$ & 1.4 & $(0.9-2.3)$ \\
\hline Never cigarette use & $7.0^{\mathrm{a}, \mathrm{b}, \mathrm{d}}$ & $(6.0-8.2)$ & $4.6^{d}$ & $(3.8-5.5)$ & $0.9^{b, c, d}$ & $(0.6-1.3)$ \\
\hline Never ENDS use & $23.8^{a, e, f, g}$ & $(22.1-25.7)$ & $4.6^{9}$ & $(3.8-5.5)$ & $0.8^{\mathrm{e}, \mathrm{f}, \mathrm{g}}$ & $(0.5-1.1)$ \\
\hline Never hookah use & $9.8^{b, e, h, i}$ & $(8.6-11.1)$ & $5.0^{i}$ & $(4.2-5.9)$ & $0.2 t^{b, e}$ & $(0.1-0.3)$ \\
\hline Never cigar use & $7.3^{f, h, j}$ & $(6.4-8.4)$ & $4.1^{\mathrm{j}}$ & $(3.4-5.0)$ & $0.2 \dagger^{c, f}$ & $(0.1-0.4)$ \\
\hline Never smokeless use & $1.4^{\mathrm{d}, \mathrm{g}, \mathrm{ij} j}$ & $(1.1-1.8)$ & $0.8^{\mathrm{d}, \mathrm{g}, \mathrm{i}, \mathrm{j}}$ & $(0.6-1.0)$ & $0.3^{\mathrm{d}, \mathrm{g}}$ & $(0.2-0.4)$ \\
\hline Adults 25+ & Weighted \% & $95 \% \mathrm{Cl}$ & Weighted \% & $95 \% \mathrm{Cl}$ & Weighted \% & $95 \% \mathrm{Cl}$ \\
\hline Never tobacco use & 2.2 & $(1.7-2.9)$ & 1.4 & $(1.0-1.9)$ & $0.3 t$ & $(0.1-0.6)$ \\
\hline Never cigarette use & $1.2^{\mathrm{a}, \mathrm{c}, \mathrm{d}}$ & $(0.9-1.6)$ & $0.9^{\mathrm{a}, \mathrm{d}}$ & $(0.6-1.3)$ & $0.2+$ & $(0.1-0.5)$ \\
\hline Never ENDS use & $5.5^{\mathrm{a}, \mathrm{e}, \mathrm{f}, \mathrm{g}}$ & $(5.1-5.9)$ & $1.4^{\mathrm{a}, \mathrm{e}, \mathrm{g}}$ & $(1.2-1.7)$ & $0.4^{\mathrm{e}, \mathrm{f}, \mathrm{g}}$ & $(0.3-0.5)$ \\
\hline Never hookah use & $0.8^{\mathrm{e}, \mathrm{h}, \mathrm{i}}$ & $(0.7-1.0)$ & $0.4^{\mathrm{e}, \mathrm{h}}$ & $(0.3-0.5)$ & $0.0 t^{e, h}$ & $(0.0-0.1)$ \\
\hline Never cigar use & $2.0^{c, f, h, j}$ & $(1.7-2.3)$ & $1.3^{\mathrm{h}, \mathrm{j}}$ & $(1.1-1.6)$ & $0.2^{\mathrm{fhh}}$ & $(0.1-0.3)$ \\
\hline Never smokeless use & $0.3^{\mathrm{d}, \mathrm{g}, \mathrm{ij}}$ & $(0.2-0.4)$ & $0.3^{\mathrm{d}, \mathrm{g}, \mathrm{j}}$ & $(0.2-0.4)$ & $0.0 t^{9}$ & $(0.0-0.1)$ \\
\hline
\end{tabular}

W1 total group unweighted denominators (P12M initiation/P30D initiation/Frequent P30D initiation):

Never tobacco use - youth (ages 12-17): 8,913/8,840/8,799; young adults (ages 18-24): 1,466/1,459/1,451; adults 25+ (ages 25 and older): 2,247/2,247/2,243

Never cigarette use - youth: 10,383/10,379/10,372; young adults: 2,584/2,584/2,569; adults 25+: 3,190/3,191/3,182

Never ENDS use - youth: 10,676/10,62110,605; young adults: 4,195/4,139/4,141; adults 25+: 12,799/12,636/12,720

Never hookah use - youth: 11,101/11,093/11,067; young adults: 3,304/3,304/3,304; adults 25+: 14,543/14,549/14,543

Never cigar use - youth: 10,775/10,748/10,741; young adults: $3,153 / 3,155 / 3,120$; adults $25+: 8,339 / 8,351 / 8,308$

Never smokeless use - youth: 11,151/11,148/11,144; young adults: 5,783/5,786/5,777; adults 25+: 14,560/14,583/14,546

Analysis included youth, young adult, and adult 25+ W1 never tobacco or tobacco product users with data at W1 and W2. Respondent age was calculated based on age at W1.

W2 single-wave weights were used to calculate estimates.

Never tobacco/product initiation is defined as not having used any tobacco or the specific product, even once or twice in lifetime.

1) P12M initiation: Defined as a never user at W1 who used any tobacco or the specific product within the past 12 months at W2.

2) P30D initiation (a subset of P12M initiation): Defined as a never user at W1 who used any tobacco or the specific product within the past 30 days at W2.

3) Frequent P30D initiation (a subset of P30D initiation): Defined as a never user at W1 who used any tobacco or the specific product within the past 30 days on at least 20 or more days at W2.

All significance tests were done between product initiations only (not any tobacco initation).

adenotes significant difference at $p<0.005$ (Bonferroni corrected for ten comparisons) between cigarette and ENDS

bdenotes significant difference at $p<0.005$ (Bonferroni corrected for ten comparisons) between cigarette and hookah

'denotes significant difference at $p<0.005$ (Bonferroni corrected for ten comparisons) between cigarette and cigar

denotes significant difference at $p<0.005$ (Bonferroni corrected for ten comparisons) between cigarette and smokeless

edenotes significant difference at $p<0.005$ (Bonferroni corrected for ten comparisons) between ENDS and hookah

fdenotes significant difference at $p<0.005$ (Bonferroni corrected for ten comparisons) between ENDS and cigar

${ }^{9}$ denotes significant difference at $p<0.005$ (Bonferroni corrected for ten comparisons) between ENDS and smokeless

h denotes significant difference at $p<0.005$ (Bonferroni corrected for ten comparisons) between hookah and cigar

idenotes significant difference at $p<0.005$ (Bonferroni corrected for ten comparisons) between hookah and smokeless

${ }^{j}$ denotes significant difference at $p<0.005$ (Bonferroni corrected for ten comparisons) between cigar and smokeless

*Respondents were asked about "e-cigarettes" at W1 and "e-products" (i.e., e-cigarettes, e-cigars, e-pipes, and e-hookah) at W2 and Wave 3.

The logit-transformation method was used to calculate the $95 \% \mathrm{Cls}$.

tEstimate should be interpreted with caution because it has low statistical precision. It is based on a denominator sample size of less than 50, or the coefficient of variation of the estimate or its complement is larger than $30 \%$.

Analyses were run on the W1 and W2 Public Use Files (https://doi.org/10.3886/ICPSR36498.v8).

$\mathrm{Cl}$, confidence interval; ENDS, electronic nicotine delivery system; P30D, past 30-day; P12M, past 12-month; W1, Wave 1; W2, Wave 2.

Table 1c Weighted Percentage of Tobacco and Product-Specific Initiation at W3 Among W2 Never Users

$$
\text { W3 (2015/16) }
$$

\begin{tabular}{|c|c|c|c|c|c|c|}
\hline \multirow{3}{*}{$\begin{array}{l}\text { W2 (2014/15) } \\
\text { Youth }\end{array}$} & \multirow{2}{*}{\multicolumn{2}{|c|}{ P12M Initiation }} & & & & \\
\hline & & & \multicolumn{2}{|c|}{ P30D Initiation } & \multicolumn{2}{|c|}{ Frequent P30D Initiation } \\
\hline & Weighted \% & $95 \% \mathrm{Cl}$ & Weighted \% & $95 \% \mathrm{Cl}$ & Weighted \% & $95 \% \mathrm{Cl}$ \\
\hline Never tobacco use & 8.9 & $(8.3-9.6)$ & 3.6 & $(3.1-4.0)$ & 0.6 & $(0.5-0.8)$ \\
\hline Never cigarette use & $4.2^{\mathrm{a}, \mathrm{b}, \mathrm{d}}$ & $(3.8-4.6)$ & $1.9^{a, b, d}$ & $(1.6-2.3)$ & $0.2^{b, c}$ & $(0.2-0.3)$ \\
\hline Never ENDS* use & $6.5^{\mathrm{a}, \mathrm{e}, \mathrm{fg}}$ & $(6.0-7.1)$ & $2.7^{\mathrm{a}, \mathrm{e}, \mathrm{f}, \mathrm{g}}$ & $(2.3-3.1)$ & $0.4^{\mathrm{e}, \mathrm{fg}}$ & $(0.3-0.6)$ \\
\hline Never hookah use & $2.6^{b, e, h, i}$ & $(2.4-3.0)$ & $0.9^{b, e, h}$ & $(0.7-1.1)$ & $0.1 t^{b, e}$ & $(0.0-0.1)$ \\
\hline
\end{tabular}


Table 1c Continued

\begin{tabular}{|c|c|c|c|c|c|c|}
\hline \multirow{3}{*}{$\begin{array}{l}\text { W2 (2014/15) } \\
\text { Youth }\end{array}$} & \multicolumn{6}{|l|}{ W3 $(2015 / 16)$} \\
\hline & \multicolumn{2}{|c|}{ P12M Initiation } & \multicolumn{2}{|c|}{ P30D Initiation } & \multicolumn{2}{|c|}{ Frequent P30D Initiation } \\
\hline & Weighted \% & $95 \% \mathrm{Cl}$ & Weighted \% & $95 \% \mathrm{Cl}$ & Weighted \% & $95 \% \mathrm{Cl}$ \\
\hline Never smokeless use & $1.7^{\mathrm{d}, g, i, j}$ & $(1.5-2.0)$ & $0.7^{\mathrm{d}, g, j}$ & $(0.6-0.9)$ & $0.2^{g, j}$ & $(0.1-0.3)$ \\
\hline Young adults & Weighted \% & $95 \% \mathrm{Cl}$ & Weighted \% & $95 \% \mathrm{Cl}$ & Weighted \% & $95 \% \mathrm{Cl}$ \\
\hline Never tobacco use & 11.4 & $(9.5-13.6)$ & 6.3 & $(5.1-7.8)$ & 0.6 & $(0.3-1.0)$ \\
\hline Never ENDS use & $6.2^{9}$ & $(5.5-7.1)$ & $3.6^{9}$ & $(2.9-4.4)$ & $0.2 \dagger$ & $(0.1-0.5)$ \\
\hline Never hookah use & $6.3^{i}$ & $(5.3-7.5)$ & $2.4^{i}$ & $(1.9-3.2)$ & $0.1 \dagger$ & $(0.0-0.2)$ \\
\hline Never cigar use & $5.8^{j}$ & $(5.1-6.6)$ & $3.6^{\mathrm{j}}$ & $(3.0-4.4)$ & $0.2 \dagger$ & $(0.1-0.4)$ \\
\hline Never smokeless use & $1.4^{\mathrm{d}, \mathrm{g}, \mathrm{i}, \mathrm{j}}$ & $(1.1-1.8)$ & $0.8^{\mathrm{d}, \mathrm{g}, \mathrm{i}, \mathrm{j}}$ & $(0.6-1.1)$ & $0.1 \dagger$ & $(0.1-0.3)$ \\
\hline Adults $25+$ & Weighted \% & $95 \% \mathrm{Cl}$ & Weighted \% & $95 \% \mathrm{Cl}$ & Weighted \% & $95 \% \mathrm{Cl}$ \\
\hline Never tobacco use & 1.8 & $(1.2-2.8)$ & 1.3 & $(0.7-2.2)$ & $0.2 t$ & $(0.0-0.6)$ \\
\hline Never cigar use & $1.4^{\mathrm{h}, \mathrm{j}}$ & $(1.1-1.7)$ & $0.9^{h, j}$ & $(0.7-1.1)$ & $0.2+$ & $(0.1-0.3)$ \\
\hline Never smokeless use & $0.4^{\mathrm{d}, \mathrm{g}, \mathrm{j}}$ & $(0.3-0.5)$ & $0.3^{g . j}$ & $(0.2-0.4)$ & $0.0 \dagger^{9}$ & $(0.0-0.1)$ \\
\hline
\end{tabular}

W2 total group unweighted denominators (P12M initiation/P30D initiation/Frequent P30D initiation):

Never tobacco use - youth (ages 12-17): 8,087/8,064/8,039; young adults (ages 18-24): 1,680/1,683/1,669; adults 25+ (ages 25 and older): 2,111/2,116/2,111

Never cigarette use - youth: 9,954/9,950/9,945; young adults: 2,985/2,986/2,979; adults 25+: 3,083/3,085/3,081

Never ENDS use - youth: 9,651/9,643/9,629; young adults: 3,715/3,716/3,701; adults $25+: 10,870 / 10,879 / 10,836$

Never hookah use - youth: 10,520/10,520/10,507; young adults: 3,395/3,397/3,394; adults 25+: 13,221/13,228/13,216

Never cigar use - youth: 10,267/10,251/10,241; young adults: $3,377 / 3,380 / 3,349$; adults $25+: 7,570 / 7,579 / 7,548$

Never smokeless use - youth: 10,497/10,496/10,490; young adults: 5,825/5,829/5,819; adults 25+: 13,749/13,771/13,741

Analysis included youth, young adult, and adult 25+ W2 never tobacco or tobacco product users with data at W2 and W3. Respondent age was calculated based on age at W2.

W3 longitudinal (all-waves) weights were used to calculate estimates.

Never tobacco/product initiation is defined as not having used any tobacco or the specific product, even once or twice in lifetime.

1) P12M initiation: Defined as a never user at W2 who used any tobacco or the specific product within the past 12 months at W3.

2) P30D initiation (a subset of P12M initiation): Defined as a never user at W2 who used any tobacco or the specific product within the past 30 days at W3.

3) Frequent P30D initiation (a subset of P30D initiation): Defined as a never user at W2 who used any tobacco or the specific product within the past 30 days on at least 20 or more days at $\mathrm{W} 3$.

All significance tests were done between product initiations only (not any tobacco initation).

${ }^{a}$ denotes significant difference at $p<0.005$ (Bonferroni corrected for ten comparisons) between cigarette and ENDS

${ }^{b}$ denotes significant difference at $p<0.005$ (Bonferroni corrected for ten comparisons) between cigarette and hookah

'denotes significant difference at $p<0.005$ (Bonferroni corrected for ten comparisons) between cigarette and cigar

denotes significant difference at $p<0.005$ (Bonferroni corrected for ten comparisons) between cigarette and smokeless

edenotes significant difference at $p<0.005$ (Bonferroni corrected for ten comparisons) between ENDS and hookah

fdenotes significant difference at $p<0.005$ (Bonferroni corrected for ten comparisons) between ENDS and cigar

${ }^{g}$ denotes significant difference at $p<0.005$ (Bonferroni corrected for ten comparisons) between ENDS and smokeless

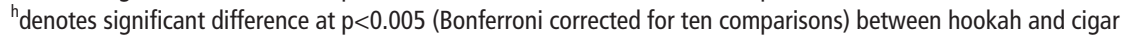

'denotes significant difference at $p<0.005$ (Bonferroni corrected for ten comparisons) between hookah and smokeless

'denotes significant difference at $\mathrm{p}<0.005$ (Bonferroni corrected for ten comparisons) between cigar and smokeless

*Respondents were asked about "e-cigarettes" at Wave 1 and "e-products" (i.e., e-cigarettes, e-cigars, e-pipes, and e-hookah) at W2 and W3.

The logit-transformation method was used to calculate the $95 \%$ Cls.

tEstimate should be interpreted with caution because it has low statistical precision. It is based on a denominator sample size of less than 50, or the coefficient of variation of the estimate or its complement is larger than $30 \%$.

Analyses were run on the W2 and W3 Public Use Files (https://doi.org/10.3886/ICPSR36498.v8).

$\mathrm{Cl}$, confidence interval; ENDS, electronic nicotine delivery system; P30D, past 30-day; P12M, past 12-month; W1, Wave 1; W2, Wave 2; W3, Wave 3.

in the percentage of never tobacco users within each age group, except an increase in never tobacco among youth between W2 and W3. Estimates of never tobacco use by product among youth, young adults and adults $25+$ are presented in online supplementary figure 3 .

\section{Longitudinal weighted pathways}

\section{P12M, P30D and frequent P30D initiation among W1 never users} across two waves

Table 1a presents, undefined by age group, any tobacco initiation and product-specific initiation across 2 years CA (at W2 or W3) among W1 never tobacco users. Among W1 never tobacco users,
$24.4 \%$ (95\% CI 23.4 to 25.4 ) of youth, $22.8 \%$ (95\% CI 20.0 to 26.0 ) of young adults, and $4.0 \%$ (95\% CI 3.1 to 5.0) of adults $25+$ started using any tobacco product at W2 or W3.

ENDS had the highest proportion of P12M initiation from W1 to W3 for youth (22.7\% (95\% CI 21.6 to 23.7$)$ ), young adults (28.4\% (95\% CI 26.5 to 30.4$)$ ), and adults $25+(6.7 \%$ (95\% CI 6.3 to 7.2 )) compared to $\mathrm{P} 12 \mathrm{M}$ initiation rates of all other products. Across all age groups, P12M initiation was the lowest for smokeless tobacco compared to the other four tobacco products.

Compared to P12M initiation, the percentage of P30D initiation across all specific tobacco products had less variability in each age group ranging from $1.8 \%$ to $6.4 \%$ among youth, $1.4 \%$ 
to $7.4 \%$ among young adults and $0.5 \%$ to $2.3 \%$ among adults $25+$. Similar to P12M initiation rates, ENDS had the highest proportion of P30D initiation from W1 to W3 compared to P30D initiation rates of all other products among youth $(6.4 \%$ (95\% CI 5.8 to 7.1$)$ ) and young adults $(7.4 \%$ (95\% CI 6.4 to 8.4)). Frequent P30D initiation for youth, young adults and adults $25+$ was rare with rates equal to or less than $1.1 \%$ for each of the specific tobacco products.

\section{W1-W2 and W2-W3 initiation, P30D initiation and frequent P30D initiation among $\mathrm{W} 1$ never users}

Examining tobacco product-specific initiation over each of the 1-year intervals (table $1 \mathrm{~b}$ and 1c) for each of the five tobacco products, ENDS had the highest proportion of W2 P12M initiation at W1-W2 for each age group (youth: 15.8\% (95\% CI 15.0 to 16.7 ); young adults: $23.8 \%$ (95\% CI 22.1 to 25.7 ); adults $25+: 5.5 \%$ (95\% CI 5.1 to 5.9$)$ ). In youth, P30D specific product initiation was $2 \%-3 \%$, except for smokeless tobacco $(<1 \%)$ and frequent P30D initiation was less than 1\% for all the products. In young adults (table $1 \mathrm{~b}$ ), P12M W2 ENDS initiation was the highest (23.8\% (95\% CI 22.1 to 25.7$)$ ) compared to other products such as hookah $(9.8 \%$ (95\% CI 8.6 to 11.1$)$ ), cigars $(7.3 \%$ (95\% CI 6.4 to 8.4$)$ ), cigarettes (7.0\% (95\% CI 6.0 to 8.2$)$ ) and smokeless tobacco (1.4\% (95\% CI 1.1 to 1.8$))$. Similarly, in adults $25+, \mathrm{P} 12 \mathrm{M} \mathrm{W} 2$ ENDS initiation was the highest (5.5\% (95\% CI 5.1 to 5.9)) compared to hookah $(0.8 \%(95 \%$ CI 0.7 to 1.0$)$ ), cigars $(2.0 \%$ (95\% CI 1.7 to 2.3$)$ ), cigarettes (1.2\% (95\% CI 0.9 to 1.6$))$ and smokeless tobacco $(0.3 \%(95 \%$ CI 0.2 to 0.4$)$ ). In young adults, W2 P30D initiation of specific tobacco products was approximately $4 \%-5 \%$ except for smokeless tobacco $(0.8 \%)$, and frequent P30D initiation of specific products was $<1 \%$. In adults $25+$, less than $2 \%$ of adults $25+$ initiated a specific tobacco product in the past 30 days, and less than $1 \%$ initiated frequent P30D use of a tobacco product.

Examining 1 year initiation rates between W2 and W3 (table 1c), the differences between the products were less pronounced. In youth, ENDS had 6.5\% (95\% CI 6.0\% to 7.1\%) W3 P12M initiation compared with cigarette $(4.2 \%$ (95\% CI 3.8 to 4.6$)$ ), cigar (3.6\% (95\% CI 3.2 to 4.0$)$ ), hookah $(2.6 \%$ (95\% CI 2.4 to 3.0$)$ ) and smokeless tobacco (1.7\% (95\% CI 1.5 to 2.0$)$ ). In young adults, P12M W3 initiation of each product was approximately 5\%-6\%, although smokeless tobacco was much lower than other products at $1.4 \%$ (95\% CI 1.1 to 1.8 ). In young adults, P30D initiation of each product was approximately 2\%-4\%, except for smokeless tobacco $(0.8 \%)$ and W3 frequent P30D initiation was less than $1 \%$. In adults $25+, \mathrm{P} 12 \mathrm{M}$ W2-W3 initiation of cigarettes, ENDS and cigars was approximately $1 \%-2 \%$, with hookah and smokeless tobacco initiated by less than 1\%. W3, P30D initiation and frequent P30D initiation were both less than $1 \%$.

W1-W2-W3 initiation pathways of exclusive and polytobacco P30D tobacco use among W1 never tobacco users

Online supplementary figure 2 provides an overview of the possible transitions in product use across W2 and W3 among W1 never tobacco users. The detailed pathways of the transitions are shown in online supplementary table 1 . Table 2 provides aggregated transitions of exclusive or polytobacco any tobacco P30D initiation rates by age group. Across all ages, more W1 adults $25+$ never users remained never users across all three waves compared with youth and young adults (youth, 89.3\% (95\% CI 88.4 to 90.1 ); young adults, $86.6 \%$ (95\% CI 83.9 to 88.9); adults $25+, 97.3 \%$ (95\% CI 96.3 to 98.0$)$ ).
W3 exclusive and polytobacco use among the subsample of W2 P30D initiators of ends or cigarettes

Table $3 \mathrm{a}$ and $3 \mathrm{~b}$ show results from a subsample of W2 initiators of ENDS or cigarettes, by age group. Among W2 youth P30D exclusive ENDS initiators (table 3a), transitioning to no tobacco use at W3 was the most common pathway $(59.0 \%$ (95\% CI 48.4 to 68.8$)$ ). Among youth who initiated P30D exclusive cigarette smoking at W2 (table $3 \mathrm{~b}$ ), no tobacco use at W3 was also the most common pathway $(40.3 \%$ (95\% CI 28.7 to 53.1$)$ ). About $20 \%$ of W1 youth never tobacco users fell into the pathway of W2 exclusive ENDS initiators who remained exclusive ENDS users at W3 (table 3a, row 2). Similarly, about $19 \%$ of W1 youth never tobacco users fell into the pathway of W2 exclusive cigarette initiators who remained exclusive cigarette users at W3 (table 3b, row 2). About 19\% of youth who initiated with exclusive cigarette use at W2 transitioned to ENDS and cigarette use at W3 (table 3b, row 4). Youth who initiated with exclusive ENDS use at W2 and transitioned to ENDS and cigarette use at W3 (table 3a, row 4) were less common, and this estimate was flagged due to high relative standard error.

\section{DISCUSSION}

Data from the US nationally representative PATH Study revealed never any tobacco use decreased over the three waves from 2013 to 2016 for youth, young adults and adults $25+$. Decreases in never tobacco use reflect increases in initiation that were driven by initiation of ENDS, which was gaining in popularity and availability in the USA during this time period. Initiating use of traditional tobacco products like cigarettes, smokeless tobacco and cigars has remained relatively stable (eg, youth P12M initiation of cigarettes in 2014/2015=4.2\%, and in 2015/2016=4.6\%). The PATH Study instrument was updated and questions were changed from asking about e-cigarettes to asking about all ENDS products between W1 and W2 as well. P12M initiation rates from 2015 to 2016 for traditional tobacco products like cigarettes, smokeless tobacco and cigars are comparable to those reported by NSDUH in 2016 (within $\sim 1 \%$ for each age group). ${ }^{14}$

A unique strength of this analysis is that three different definitions of initiation (P12M, P30D and frequent P30D) for overall tobacco use and product specific tobacco use are reported over a 2-year period, as well as the 1-year intervals within that time span, for three different age groups. Within each age group and looking across tobacco products, the highest proportion of P12M initiation was among W1 never ENDS users who initiated ENDS use at W2. The higher rates of P12M ENDS initiation compared with other definitions may signal that a fair amount of ENDS initiation is experimental and does not persist to be identified as P30D or frequent P30D use. Across all age groups, initiation from W1 to W2 of smokeless tobacco was lower than initiation of the other products for both P12M and P30D. Initiation of ENDS from W2 to W3 was not higher than initiation of cigarettes, cigars or hookah. It is noted that these data were collected before the documented increase in use of ENDS among US youth in $2018 .^{20}$ Specifically, data collection occurred before the rapid growth of JUUL in the USA between 2016 and 2018; JUUL delivers a high dose of nicotine and therefore has a greater potential for addictiveness than many of the first generation products available in 2013-2016. ${ }^{29-32}$

Examining longitudinal three-wave pathways, most never tobacco users at W1 remained never users across all three waves. A pattern of persistent never tobacco use across the waves 


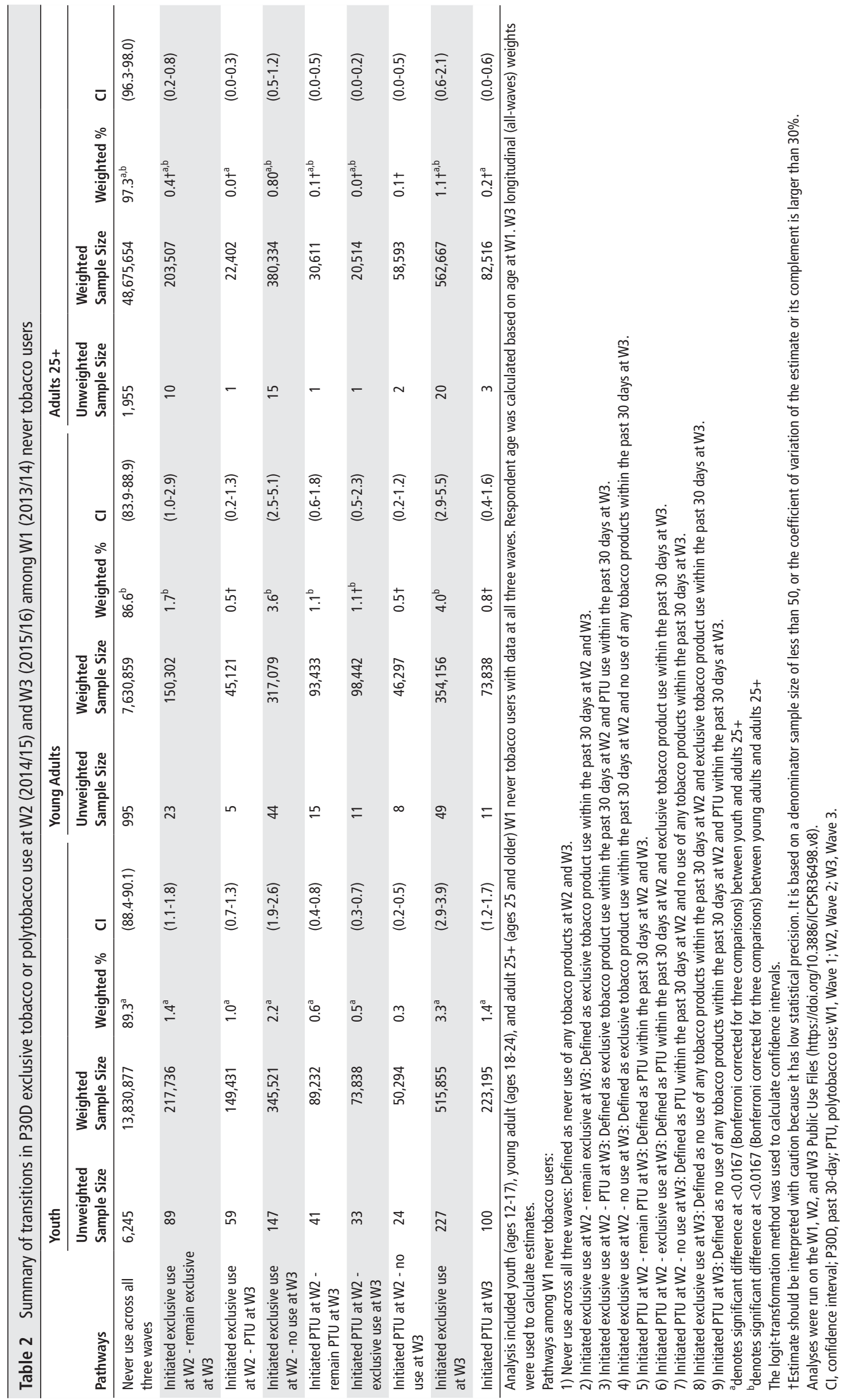




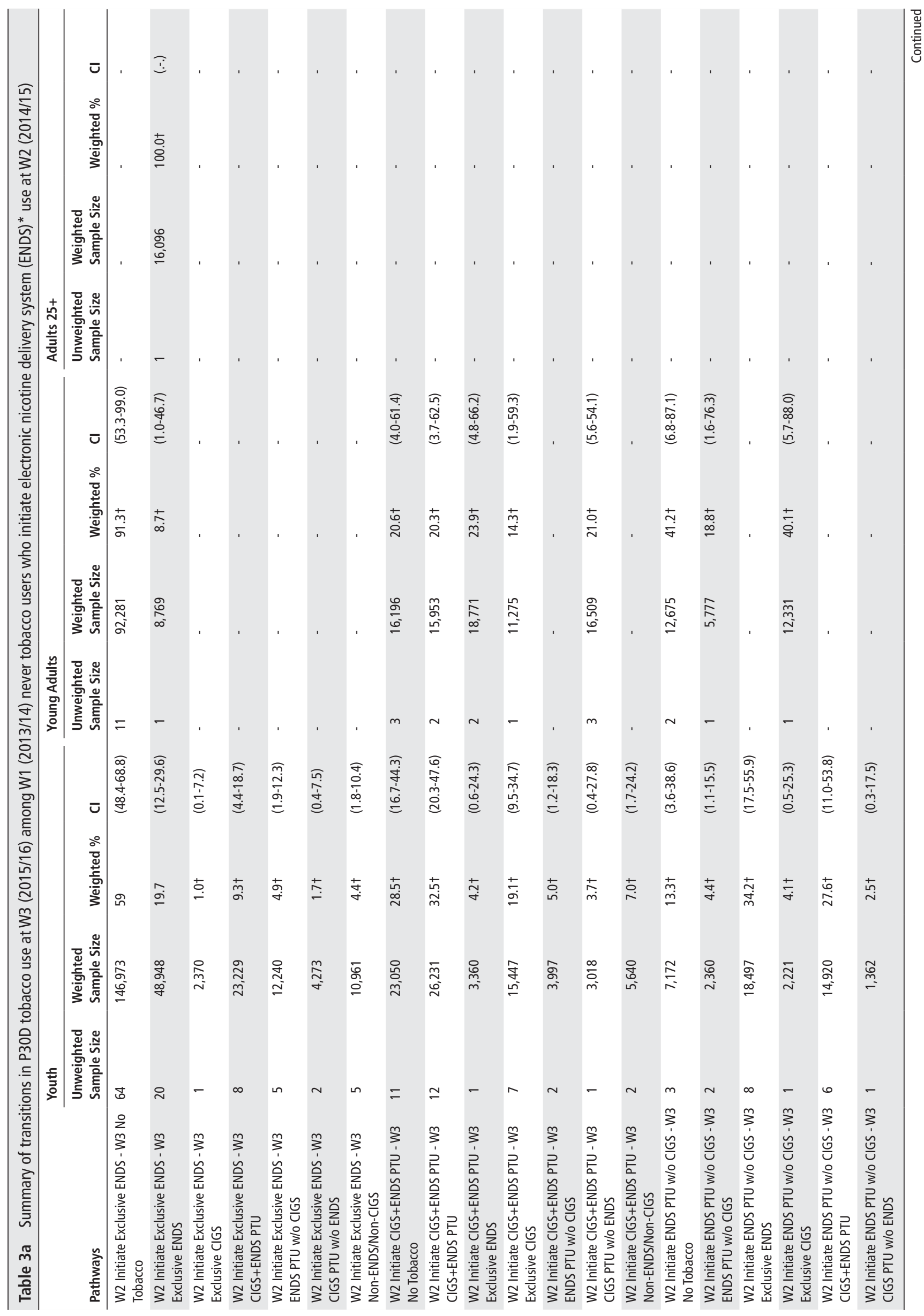

ᄋ

$\stackrel{\overrightarrow{\vec{p}}}{\overrightarrow{\mathrm{s}}}$

흫 


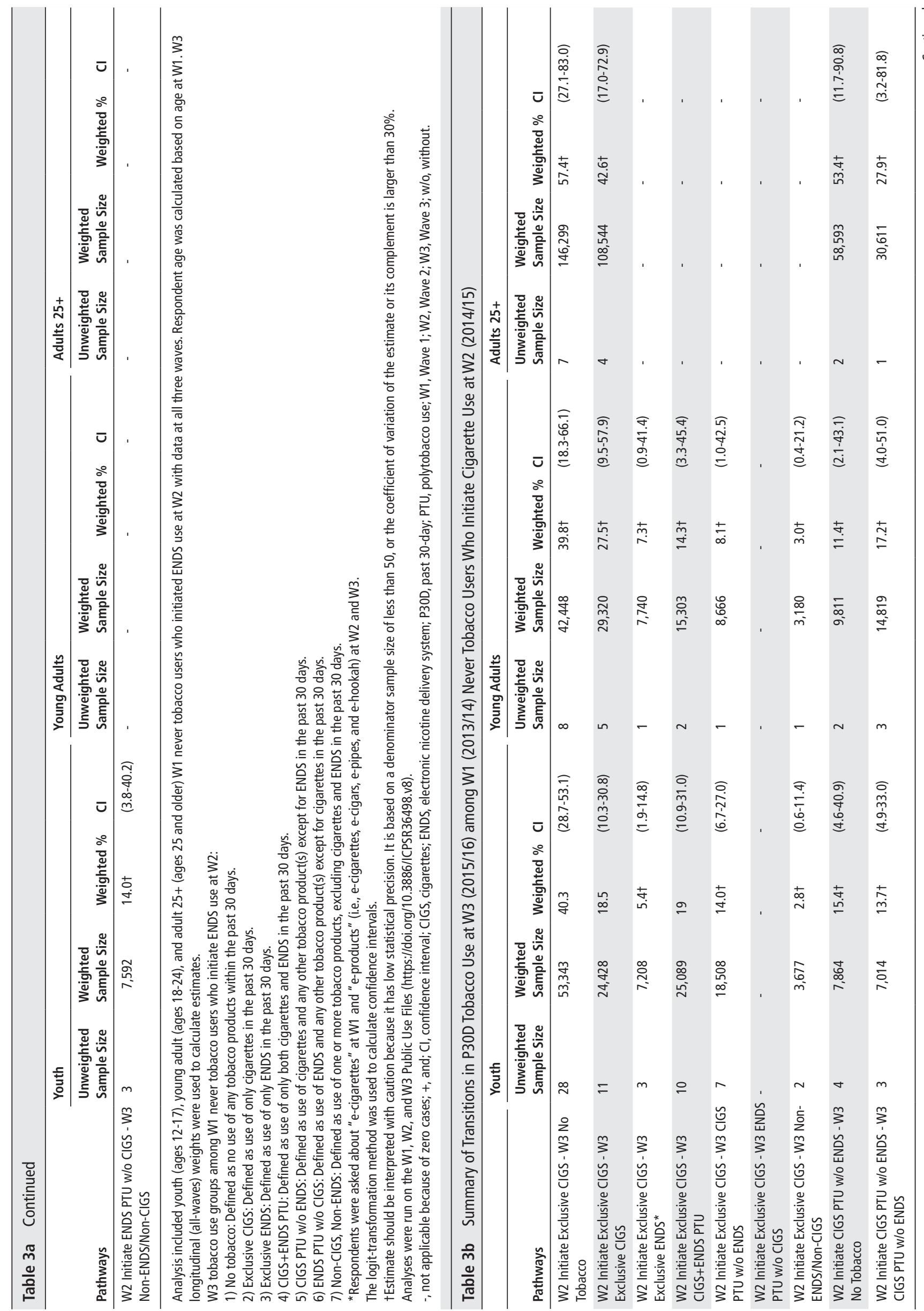









\section{What this paper adds}

- Across all age groups, never any tobacco use decreased from 2013 to 2016, reflecting overall increases in tobacco initiation in the population.

- The higher rates of past 12-month (P12M) initiation of electronic nicotine delivery systems (ENDS) may signal that a fair amount of ENDS initiation is experimental and does not persist to be identified as past 30-day (P30D) or frequent P30D use.

- Comparing exclusive and polytobacco use pathways across ages, more youth and young adults than adults 25+ initiated exclusive use of a tobacco product at W2 and then had no tobacco use at W3. More W1 adult 25+ never users remained never users across all three waves compared with youth and young adults.

- Among youth W2 P30D initiators of exclusive ENDS or cigarettes, the most common W3 outcome was not using any tobacco.

was highest among adults $25+$, for whom tobacco abstinence may be an established behaviour. Findings of persistent never use across the three age groups mirror other recent studies of tobacco use transitions. ${ }^{33} 34 \mathrm{P} 30 \mathrm{D}$ exclusive product initiation at W2 or W3 was more common than P30D polytobacco initiation across all age groups. A higher proportion of W2 exclusive initiators stopped using tobacco at W3 compared with W2 initiators who were polytobacco users. Initiating use of multiple tobacco products within the same time period may be a risk factor for continued use over time. Given the growing phenomenon of concurrent multiple tobacco product use, ${ }^{35-40}$ prevention strategies could include discussion of how combining use of tobacco products may increase risk of addiction. Kasza et al ${ }^{41}$ examined predictors of ever or P30D tobacco initiation over a 1 -year period and found that after controlling for demographics, ever use of another type of tobacco product was a significant predictor of tobacco product initiation.

Given growing evidence that ENDS initiation is associated with subsequent cigarette initiation, ${ }^{7} 10$ 20-24 analyses that describe specific ENDS and cigarette pathways across the three waves for each age group were explored. Among youth, $59.0 \%$ of W2 exclusive ENDS initiators were not using any tobacco at W3, whereas $40.3 \%$ of W2 exclusive cigarette initiators were not using any tobacco at W3. It is noted that approximately $20 \%$ of youth who initiated exclusively with ENDS at W2 were also exclusive ENDS users at W3 during this time period of 2013-2016 compared with 18.5\% of youth who initiated exclusively with cigarettes and remained exclusive cigarette users at W3. As accessibility and trial of ENDS products among youth in the USA increased from 2017 to $2019,{ }^{81730}$ it remains to be studied what proportion of ENDS initiators remain exclusive ENDS users.

While $19 \%$ of youth exclusive cigarette initiators become dual ENDS and cigarette polytobacco users a year later, that same pathway among youth who initiate with ENDS is flagged due to low sample sizes. These observations add to the growing evidence base that traditional tobacco products, such as cigarette use $^{42}$ and smokeless tobacco use, ${ }^{43}$ may be more likely to be persistent over time compared with other tobacco products such as ENDS, cigars and hookah. ${ }^{44-46}$

\section{Limitations}

Limitations of this report include recall bias from a self-report study questionnaire. Additionally, the PATH Study asked about 'e-cigarettes' at W1 and 'e-products' (ie, e-cigarettes, e-cigars, e-pipes and e-hookah) at W2 and W3. This approach may have resulted in misclassification of ENDS-specific use between W1 and the subsequent waves. It is noted that this report presents prevalence rates unadjusted for demographic or behavioural variables, which may be important variables to consider in future analyses. It is also noted that there may be variability in initiation rates compared with other published PATH Study papers due to differences in definitions or those included in the analytic sample. Weighted longitudinal analyses over the follow-up period excluded participants who were missing data at one of the waves. The extent of missing data and the small number of observations for specific low-prevalence pathways limit interpretation.

\section{Summary and implications}

This report provides a unique examination of rates of never use across 3 years, three different definitions of initiation rates of tobacco overall and product-specific use, and three-wave longitudinal pathways that capture product initiation at W2 and their transitions at W3. Despite growing rates of P12M ENDS initiation in the USA, youth ENDS P30D initiation and frequent P30D initiation are less common. Among W2 P30D initiators of exclusive ENDS or cigarettes, the most common W3 outcome is not using any tobacco. While ENDS initiation rates need to be monitored and addressed in prevention efforts, these data suggest that we must also remain vigilant and maintain a strong public health focus on prevention of cigarette smoking initiation as well as initiation of other tobacco products.

Contributors CAS and ES led the conceptual design. CAS drafted initial manuscript and all authors critically revised it. ELS and MJH conducted statistical analysis and all authors contributed to interpretation of results. All authors approved the work for journal publication and agree to be accountable for all aspects of the work.

Funding This manuscript is supported with Federal funds from the National Institute on Drug Abuse, National Institutes of Health, and the Center for Tobacco Products, Food and Drug Administration, Department of Health and Human Services, under a contract to Westat (Contract No. HHSN271201100027C).

Disclaimer The findings and conclusions in this report are those of the authors and do not necessarily represent the official position of the US Department of Health and Human Services or any of its affiliated institutions or agencies.

Competing interests WMC reports long-term stock holdings in General Electric Company, 3M Company, and Pfizer Incorporated, unrelated to this manuscript. No financial disclosures were reported by the other authors of this paper.

\section{Patient consent for publication Not required.}

Ethics approval The study was conducted by Westat and approved by the Westat Institutional Review Board. All participants ages 18 and older provided informed consent, with youth participants ages 12 to 17 providing assent while their parent/ legal guardian provided consent.

Provenance and peer review Not commissioned; externally peer reviewed.

Data availability statement Data from the PATH Study Wave 1 to Wave 3 are available for download as Public Use Files in a public, open access repository (https://www.icpsr.umich.edu/icpsrweb/NAHDAP/studies/36498). Conditions of use are available at the website above.

\section{ORCID iDs}

Cassandra A Stanton http://orcid.org/0000-0001-5329-6261

Eva Sharma http://orcid.org/0000-0002-1328-508X

Karin A Kasza http://orcid.org/0000-0002-4492-098X

Kathryn C Edwards http://orcid.org/0000-0002-0645-1705

Michael J Halenar http://orcid.org/0000-0002-8703-3811

Kristie A Taylor http://orcid.org/0000-0001-6668-7923

Maansi Bansal-Travers http://orcid.org/0000-0002-7944-3570

Lisa D Gardner http://orcid.org/0000-0003-3340-2818 
Heather L Kimmel http://orcid.org/000-0001-8278-0095

\section{REFERENCES}

1 Miech RA, Schulenberg JE, Johnston LD, et al. Trends in lifetime prevalence of use of various drugs in grades 8, 10, and 122018.

2 Hu SS, Neff L, Agaku IT, et al. Tobacco Product Use Among Adults - United States, 2013-2014. MMWR Morb Mortal Wkly Rep 2016;65:685-91.

3 Jamal A, Gentzke A, Hu SS, et al. Tobacco Use Among Middle and High School Students - United States, 2011-2016. MMWR Morb Mortal Wkly Rep 2017:66:597-603.

4 US Department of Health and Human Services. The Health Consequences of Smoking - 50 Years of Progress: A Report of the Surgeon General. Atlanta, GA: US Department of Health and Human Services, Centers for Disease Control and Prevention, National Center for Chronic Disease Prevention and Health Promotion, Office on Smoking and Health 2014;943.

5 Lee YO, Hebert CJ, Nonnemaker JM, et al. Youth tobacco product use in the United States. Pediatrics 2015:135:409-15.

6 Wang TW, Gentzke A, Sharapova S, et al. Tobacco Product Use Among Middle and High School Students - United States, 2011-2017. MMWR Morb Mortal Wkly Rep 2018;67:629-33.

7 Berry KM, Fetterman JL, Benjamin EJ, et al. Association of electronic cigarette use with subsequent initiation of tobacco cigarettes in US youths. JAMA Netw Open 2019;2:e187794.

8 Cullen KA, Gentzke AS, Sawdey MD, et al. E-Cigarette use among youth in the United States, 2019. JAMA 2019. doi:10.1001/jama.2019.18387. [Epub ahead of print: 05 Nov 2019].

9 Kasza KA, Coleman B, Sharma E, et al. Correlates of transitions in tobacco product use by U.S. adult tobacco users between 2013-2014 and 2014-2015: findings from the path study wave 1 and wave 2. Int J Environ Res Public Health 2018;15:2556.

10 Watkins SL, Glantz SA, Chaffee BW. Association of noncigarette tobacco product use with future cigarette smoking among youth in the population assessment of tobacco and health (path) study, 2013-2015. JAMA Pediatr 2018;172:181.

11 Parker MA, Villanti AC, Quisenberry AJ, et al. Tobacco product harm perceptions and new use. Pediatrics 2018;142. doi:10.1542/peds.2018-1505. [Epub ahead of print: 05 Nov 2018].

12 Strong DR, Messer K, Hartman SJ, et al. Pre-adolescent receptivity to tobacco marketing and its relationship to acquiring friends who smoke and cigarette smoking initiation. Ann Behav Med 2017;51:730-40.

13 Sidani JE, Shensa A, Naidu MR, et al. Initiation, progression, and sustained waterpipe use: a Nationally-Representative longitudinal study of US young adults. Cancer Epidemiology and Prevention Biomarkers 2017;0687.

14 Center for Behavioral Health Statistics and Quality. National Survey on Drug Use and Health: Detailed Tables. Rockville. 2017. MD: Substance Abuse and Mental Health Services Administration, 2016

15 Berry KM, Reynolds LM, Collins JM, et al. E-Cigarette initiation and associated changes in smoking cessation and reduction: the population assessment of tobacco and health study, 2013-2015. Tob Control 2019;28:42-49.

16 Thompson AB, Mowery PD, Tebes JK, et al. Time trends in smoking onset by sex and Race/Ethnicity among adolescents and young adults: findings from the 2006-2013 national survey on drug use and health. Nicotine Tob Res 2018;20:312-20.

17 Gentzke AS, Creamer M, Cullen KA, et al. Vital Signs: Tobacco Product Use Among Middle and High School Students - United States, 2011-2018. MMWR Morb Mortal Wkly Rep 2019;68:157-64.

18 Wang TW, Asman K, Gentzke AS, et al. Tobacco Product Use Among Adults - United States, 2017. MMWR Morb Mortal Wkly Rep 2018;67:1225-32.

19 Choi SH, Stommel M. Impact of age at smoking initiation on smoking-related morbidity and all-cause mortality. Am J Prev Med 2017;53:33-41.

20 National Academies of Sciences E, and Medicine,.. Public health consequences of e-cigarettes. Washington, DC: The National Academies Press, 2018.

21 Stanton CA, Bansal-Travers M, Johnson AL, et al. Longitudinal e-cigarette and cigarette use among US youth in the path study (2013-2015. JNCl: Journal of the National Cancer Institute, 2019: 111. 1088-96.
22 Chaffee BW, Watkins SL, Glantz SA. Electronic cigarette use and progression from experimentation to established smoking. Pediatrics 2018;141:e20173594.

23 Bold KW, Kong G, Camenga DR, et al. Trajectories of e-cigarette and conventional cigarette use among youth. Pediatrics 2018;141:e20171832.

24 Barrington-Trimis JL, Urman R, Berhane K, et al. E-Cigarettes and future cigarette use. Pediatrics 2016;138:e20160379.

25 Hyland A, Ambrose BK, Conway KP, et al. Design and methods of the population assessment of tobacco and health (path) study. Tob Control 2017;26:371-8.

26 Tourangeau R, Yan T, Sun H, et al. Population assessment of tobacco and health (path) reliability and validity study: selected reliability and validity estimates. Tob Control 2019;28:663-8.

27 McCarthy PJ. Pseudoreplication: further evaluation and applications of the balanced half-sample technique 1969.

28 Judkins DR. Fay's method for variance estimation. Journal of Official Statistics 1990;6:223.

29 Willett JG, Bennett M, Hair EC, et al. Recognition, use and perceptions of JUUL among youth and young adults. Tob Control 2019;28:115-6.

30 Miech R, Johnston L, O'Malley PM, et al. Adolescent Vaping and Nicotine Use in 2017-2018 - U.S. National Estimates. N Engl J Med 2019;380:192-3.

31 Vallone DM, Bennett $\mathrm{M}, \mathrm{Xiao} \mathrm{H}$, et al. Prevalence and correlates of JUUL use among a national sample of youth and young adults. Tob Control 2019;28:603-9.

32 Hammond D, Wackowski OA, Reid JL, et al. Use of JUUL e-cigarettes among youth in the United States. Nicotine Tobacco Research 2018:66.

33 Hair EC, Romberg AR, Niaura R, et al. Longitudinal tobacco use transitions among adolescents and young adults: 2014-2016. Nicotine Tob Res 2019;21:458-68.

34 Niaura R, Rich I, Johnson AL, et al. Young adult tobacco and e-cigarette use transitions: examining stability using multistate modeling. Nicotine Tobacco Research 2019;66.

35 Stanton CA, Halenar MJ. Patterns and correlates of multiple tobacco product use in the United States. Nicotine Tob Res 2018;20:S1-4.

36 Antognoli E, Koopman Gonzalez S, Trapl E, et al. Cigarettes, little Cigars, and Cigarillos: initiation, motivation, and decision-making. Nicotine Tob Res 2018;20:S5-11.

37 Osman A, Kowitt SD, Ranney LM, et al. Trends and racial disparities in mono, dual, and poly use of tobacco products among youth. Nicotine Tob Res 2018;20:S22-30.

38 Cho J, Goldenson NI, Stone MD, et al. Characterizing polytobacco use trajectories and their associations with substance use and mental health across mid-adolescence. Nicotine Tob Res 2018;20:S31-8.

39 Sutter ME, Everhart RS, Miadich S, et al. Patterns and profiles of adolescent tobacco users: results from the Virginia youth survey. Nicotine Tob Res 2018;20:S39-47.

40 Sung $\mathrm{H}-\mathrm{Y}$, Wang $\mathrm{Y}$, Yao T, et al. Polytobacco use and nicotine dependence symptoms among US adults, 2012-2014. Nicotine Tob Res 2018;20:S88-98.

41 Kasza K, Edwards KC, Tang Z, et al. Correlates of tobacco product initiation among youth and adults in the USA: findings from the Path Study Waves 1-3 (2013-2016). Tob Control 2020:29:5191-202.

42 Taylor KA, Sharma E, Edwards KC, et al. Longitudinal pathways of exclusive and polytobacco cigarette use among youth, young adults, and adults in the USA: findings from the PATH Study Waves 1-3 (2013-2016). Tob Control 2020;29:s139-46.

43 Sharma E, Edwards KC, Halenar MJ, et al. Longitudinal pathways of exclusive and polytobacco smokeless use among youth, young adults, and adults in the USA: findings from the PATH Study Waves 1-3 (2013-2016). Tob Control 2020:29:5170-7.

44 Edwards KC, Sharma E, Halenar MJ, et al. Longitudinal pathways of exclusive and polytobacco cigar use among youth, young adults, and adults in the USA: findings from the PATH Study Waves 1-3 (2013-2016). Tob Control 2020;29:s163-9.

45 Sharma E, Bansal-Travers M, Edwards KC, et al. Longitudinal pathways of exclusive and polytobacco hookah use among youth, young adults, and adults in the USA: findings from the PATH Study Waves 1-3 (2013-2016). Tob Control 2020;29:s155-62.

46 Stanton CA, Sharma E, Edwards KC, et al. Longitudinal transitions of exclusive and polytobacco electronic nicotine delivery systems (ends) use among youth, young adults, and adults in the USA: findings from the PATH Study Waves 1-3 (2013-2016). Tob Control 2020;29:s147-54 